\title{
Finite Element Modeling of the Neuron-Electrode Interface
}

\section{A Valuable Tool for Studying and Optimizing the Neuron-Electrode Contact}

\begin{abstract}
The electrical contact between an embedded microelectrode and a cultured neuron depends on the geometry of the neuron-electrode interface. The contact is improved when the electrode is covered, or sealed, completely by the neuron. In this article, the finite element method is proposed as a tool for modeling the electrical properties of the neuron-electrode interface. This method permits numerical solutions of volume conductor problems for a variety of geometries, without prior restriction of the current paths. Simulations are focused on the influence of the geometry on the transfer of an extracellularly applied stimulus current to the neuron and on the sealing resistance. A comparison is also made between finite element modeling and lumped circuit modeling.
\end{abstract}

\section{Overview}

One of the objectives of the neurotechnolgy program in our group is to establish a neuro-electronic interface with the human nervous system on the cellular level [1]. For the development of this implantable device, a two-step strategy is followed. In the first step, a large number of electrical contacts are made between an array of microelectrodes and a layer of cultured neurons. In the second step, after implantation, synaptical contacts will be formed between the neuronal culture and the nervous system.

The electrical contact between microelectrodes and cultured neurons can be studied in detail using planar substrate multielectrode arrays (MEAs). In Fig. 1(a), one of the MEAs that is currently used by our group is depicted. It consists of a hexagonal array of 61 microelectrodes, each with a diameter of $10 \mu \mathrm{m}$. In this example, the array is covered with neuronal cells that are isolated from dorsal root ganglions of neonatal rats. In other groups, similar MEAs are widely used with increasing success for long-term recording and stimulation of neural networks in culture [2-4]. From the large

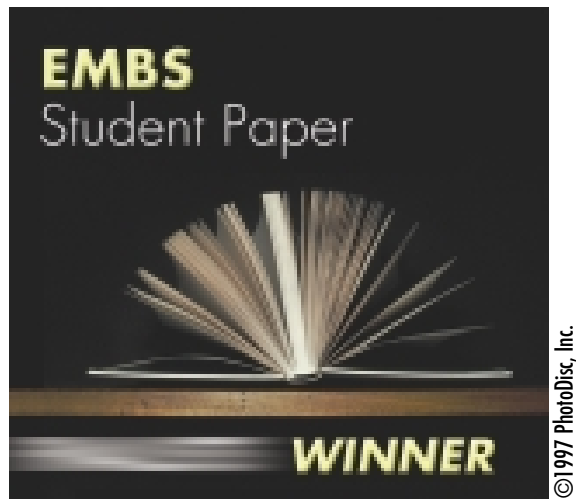

terface (not to be confused with the neuro-electronic interface). Two examples of neuron-electrode interfaces are depicted in Figs.1(b) and 1(c). Although electrodes are often covered only partially with neurons, sometimes the neurons cover the electrode completely.

When a neuron covers, or seals, an elec$\ddot{E}$ trode completely, a specific part of the interface becomes important for the neuron-electrode contact: the sealing gap between the neuronal membrane and the substrate (Fig. 2). Experimental results show that in this case the amplitude of recorded action potentials increases and the stimulation threshold decreases [6, 7]. These improvements of the contact are ascribed to the decreased resistance of the sealing gap. Hence, the electrical properties of the neuron-electrode interface are related to its geometry; i.e., size, shape and relative positions of neuron and electrode.

For interface geometries of complete sealing, it has become common practice to model the neuron-electrode contact with simple lumped circuits [5-7], based on the two major current pathways that exist in these cases: the leakage current and the current through the cell (Fig. 2). The parameters of these circuits are approximated from the interface geometry. However, the validity of this method is questionable for at least two reasons. First, major simplifications are made in the representation of the electrical properties of the interface by the definition of only two current paths. Second, it is difficult to compute the component values from the geometry, unless the represented geometry is very simple (e.g., axisymmetrical) or can be approximated by discretization into simple elements. een a neuron and an electrode. sically, this transfer is mediated by the volume conducting properties of the extracellular space - the culture medium. This volume conductor and its electrical interactions with the electrode and the neuron is the primary subject of this article: it is called the neuron-electrode in- 
gain insight into the relationship between geometry of the neuron-electrode interface and its electrical behavior. This parametrical geometry is depicted in Fig. 3 . The neuron is modeled as a circular soma of radius $r_{c}$, with a parabolic height profile $h_{c}$. The axon and dendrites of the neuron are not included in this version of the model, since we are primarily interested in situations in which neurons cover electrodes completely or partially. In each simulation, the radius of the neuron is varied from $r_{c}=7$ to $r_{c}=20 \mu \mathrm{m}$ in four steps. These values are representative of a wide variety of vertebral neuronal types, such as cortical neurons, dorsal root ganglion neurons, and spinal cord neurons [8-10]. The height of the neuron is taken as $h_{c}=5$ $\mu \mathrm{m}$, based on the range of neuronal diameters and the assumption of a certain flattening of the neuron in culture, due to its adhesion to the substrate. The neuron is positioned with an eccentricity $\mathrm{x}_{\mathrm{c}}$ on top of an electrode with radius $r_{e}=5 \mu \mathrm{m}$, which is located in the bottom of a well with a depth of $h_{e}=0.8 \mu \mathrm{m}$. A sealing gap of thickness $d_{g}=10 \mathrm{~nm}$ is modeled between the soma and the substrate, representing a strong neuron-substrate adhesion [11].

\section{Finite Element Model}

The geometry of the neuron-electrode interface is implemented in ANSYS Finite Element Software (SAS IP Inc. Houston, TX) release 5.4 [Fig. 4(a)]. The medium surrounding the interface is represented by a volume conductor of conductivity $\sigma \quad=1.65 \mathrm{~S} / \mathrm{m}$. The intracellular volume is modeled with a conductivity of $\sigma=1.43 \mathrm{~S} / \mathrm{m}$. The thickness of the sealing gap $d_{g}$ is two orders of magnitude smaller than the other geometry parameters. Implementing the sealing gap with its actual thickness would require a mesh with either impractical element shapes, or an unfeasibly large number of elements. Therefore, the sealing gap is implemented with a fixed thickness of $d \quad=0.5 \mu \mathrm{m}$, and its conductivity is compensated for the difference between modeled and implemented thickness, according to:

$$
\sigma_{\text {seal }}=\sigma_{\text {medium }} \frac{d_{g}}{d_{\text {implemented }}} .
$$

All volumes in the model are meshed and filled with tetrahedral-shaped volume elements (SOLID98), which permit numerical solutions of the Poisson equation:

$$
\vec{\nabla} \cdot(\sigma \vec{\nabla} V)=0
$$

with $V$ the electrical potential and $\sigma$ the conductivity of the culture medium, the intracellular fluid, or the fluid in the seal-

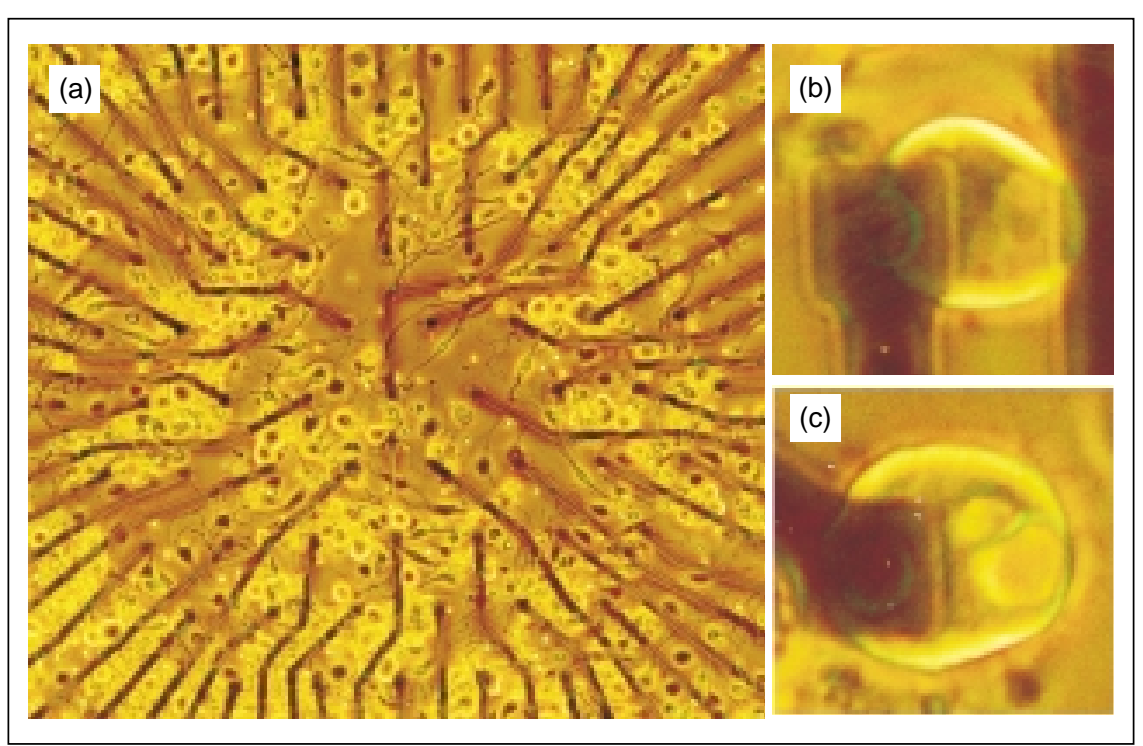

1. (a) Multielectrode array, as used in our group, with a culture of DRG neurons from neonatal rats after 1 DIV. (b) DRG neuron partially covering an electrode. (c) Complete coverage of an electrode by a DRG neuron.

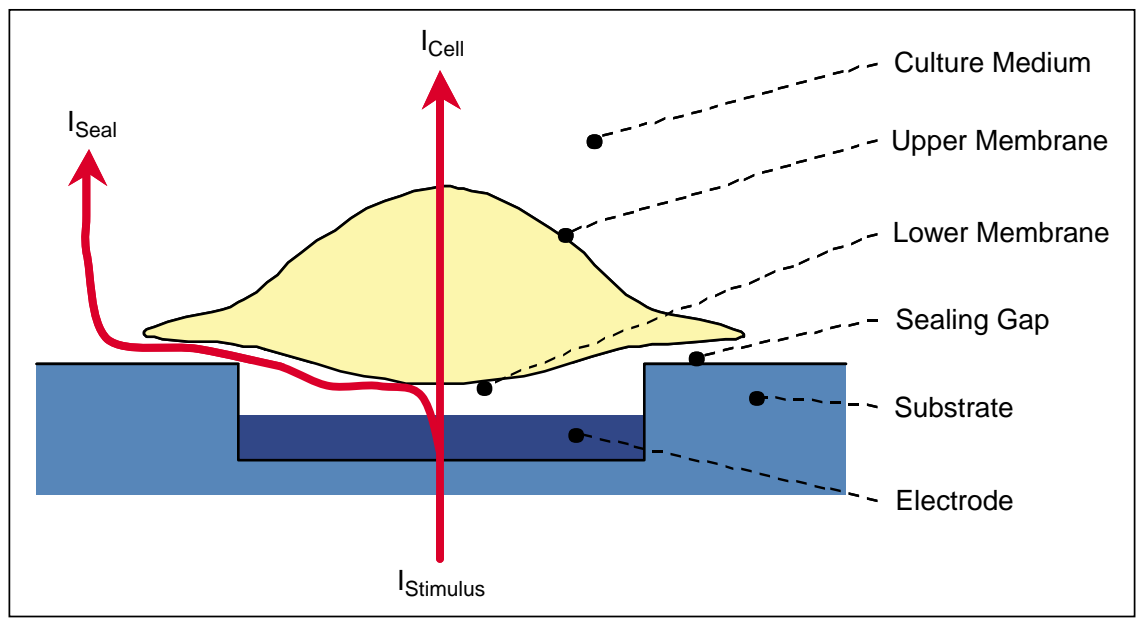

2. Schematic presentation of the neuron-electrode interface.

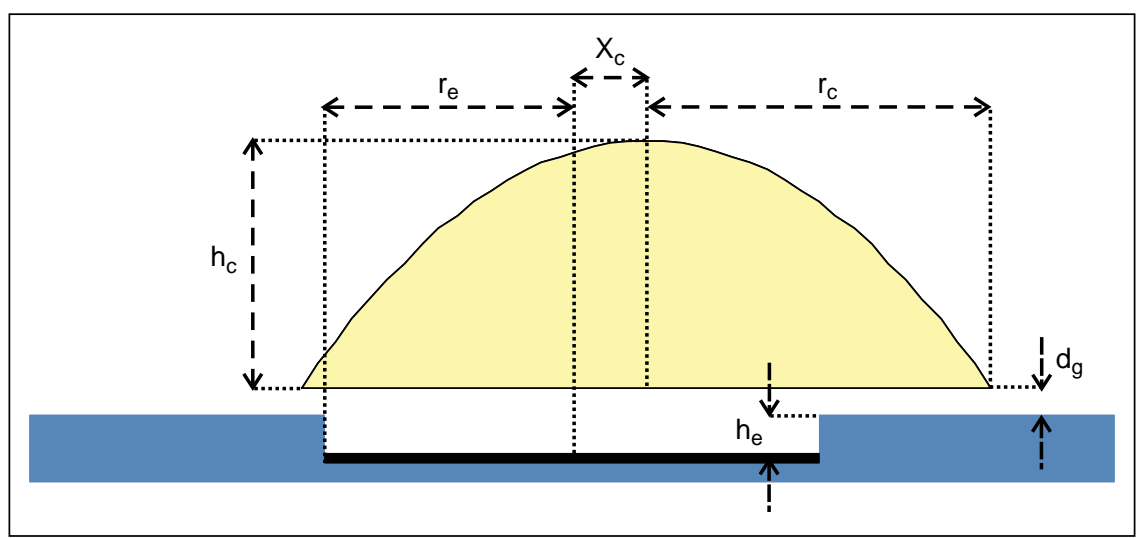

3. Parametric geometry of the neuron-electrode interface. 


\section{The electrode}

\section{impedance has no}

\section{effect on the stimulus}

\section{transfer and therefore}

\section{the electrode}

\section{impedance is not}

\section{required in this model.}

The electrical properties of the neuronal membrane are assumed to be highly anisotropic. Due to the ion channels, current can only flow in the direction perpendicular to the surface of the membrane. Hence, there is no need for modeling the membrane using volume elements, and the membrane can be represented by a set of extracellular and intracellular membrane nodes [Fig. 4(b)]. Each intracellular membrane node corresponds to one extracellular membrane node and is connected to it by a resistive circuit element. The resistance of this element is com- puted using the membrane conductivity and the membrane area represented by the connected node. Membrane capacity is left out of the model, since pilot simulations with a membrane capacity of 1 $\mu \mathrm{F} / \mathrm{cm}^{2}$ revealed that the time constant of the membrane potential is less than a microsec (results not presented). The membrane potential is computed from the potential difference between an intracellular membrane node and its corresponding extracellular membrane node. No active (e.g., Hodgkin-Huxley) properties of the membrane are modeled.

Since current stimulation is used, the electrode impedance has no effect on the stimulus transfer and therefore the electrode impedance is not required in this model. All nodes on the surface of the electrode are coupled in the voltage domain, and a stimulus current is applied to the total electrode surface. The nodes at the outer boundary of the modeled part of the culture medium are set to zero potential, representing a counter electrode that is far away from the neuron-electrode interface.

In each simulation, both the stimulus transfer and the resistance of the sealing gap are computed. The stimulus transfer is defined as the maximum depolarization of the upper membrane, due to a stimulus current of $I=1 \mathrm{nA}$ through the electrode. When the neuron completely covers the electrode, this stimulus current will depolarize the upper membrane by several millivolts. This permits easy interpretation of the results with re- spect to the stimulus threshold for eliciting an action potential.

From previous work, it is known that at complete sealing, the current through the cell is negligible compared to the current through the sealing gap. The potential at the electrode surface is therefore mainly determined by the sealing resistance, which is thereby computed as:

$$
R_{\text {seal }}=\frac{V_{e}}{I_{\text {stim }}} .
$$

When the sealing becomes defective, a sealing resistance no longer exists. Instead, the value computed from Eq. (3) must be interpreted as a spreading resistance.

\section{Results}

\section{Complete and Defect Sealing}

In practice, cultured neurons rarely cover an electrode completely. Therefore, the effects of varying eccentricity of a neuron with a radius of $r_{c}=7 \mu \mathrm{m}$ are studied using eccentricities of $x_{c}=0$ (complete sealing) and $x_{c}=9 \mu \mathrm{m}$ (defective sealing). In Fig. 5, the computed potential distribution due to a $1 \mathrm{nA}$ stimulus current is depicted in a cross section of the model. The equipotential lines in the medium are marked by the boundaries between two adjacent colored regions. At complete sealing [Fig. 5(a)], these equipotential lines are concentrated in the sealing gap, indicating a potential drop over the sealing gap. A maximum depolarization of $1.02 \mathrm{mV}$ (stimulus transfer) occurs at the upper membrane, and a hyperpolarization of 2.25 $\mathrm{mV}$ occurs at the lower membrane. When

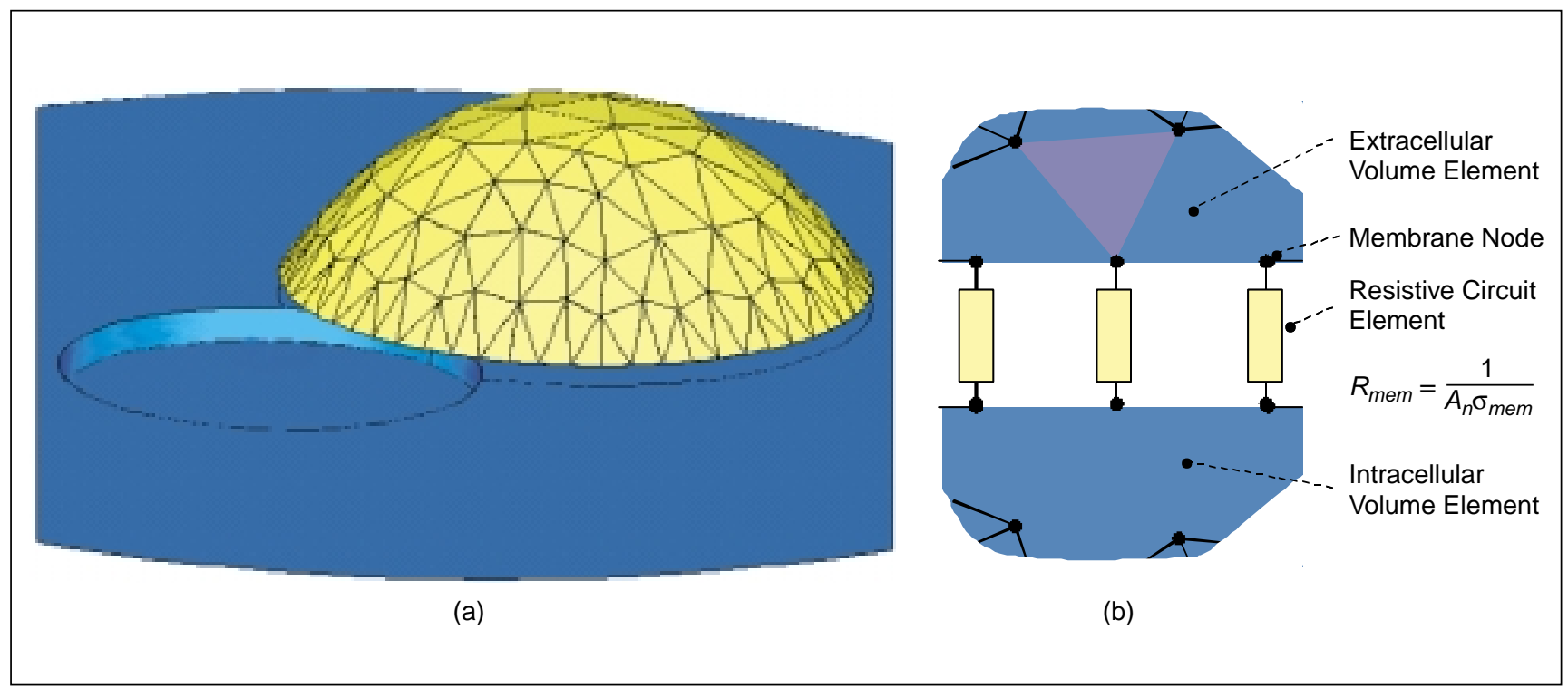

4. (a) 3-D visualization of the neuron electrode interface geometry, as implemented in the ANSYS finite element software. (b) The membrane is represented by a set of resistive circuit elements, connecting the extracellular and intracellular membrane nodes. 
the sealing is defective [Fig. 5(b)], the equipotential lines are spread up more widely, indicating a leakage current into the medium. The changes in membrane potential are reduced to the microvolt range. From the electrode potential $V_{e}$, and the stimulus current at $x_{c}=0$, a sealing resistance of $3.28 \mathrm{M} \Omega$ is computed and at $x_{c}=9 \mu \mathrm{m}$ a spreading resistance of only $36.6 \mathrm{k} \Omega$ is computed. These results reveal that sealing resistance is strongly related to the stimulus transfer. The transition from complete to defective sealing causes a drastic decrease in both the stimulus transfer and the sealing resistance.

\section{Neuron-Substrate Adhesion}

For culturing monolayers of neurons, a proper adhesion of the neurons to the substratum is desired. Often, adhesion promoters are used for this purpose, such as poly-1-lysin, laminin, and polyethyleneimine. Due to a stronger adhesion to the substrate, neurons generally will spread out and become flatter. Hence, adhesion will affect the radius and the height of the neuron. Furthermore, the electrical properties of the sealing gap can be affected. These properties are the subject of some consideration in the literature. Generally, a bulk resistivity is assumed in the gap and the thickness of the gap is assumed to be very small, which results in sealing resistances of several megohms.

In Fig. 6, the simulated effects of variations in neuronal radius and height, and also of the thickness of the sealing gap, are presented for cases of complete sealing $\left(x_{c}=0\right)$. The stimulus transfer [Fig. 6(a)] and the sealing resistance [Fig. 6(b]) are plotted versus the radius of the neuron. The solid line represents the results from simulation with normal height and thickness of the sealing gap. Both stimulus transfer and sealing resistance increase with increasing radius of the neuron. The dashed lines represent results from simulations in which one geometry parameter $\left(h_{c}\right.$ or $\left.d_{g}\right)$ is varied.

Although the height of the neuron is varied extensively to $h_{c}=1 \mu \mathrm{m}$ and to $h_{c}=9 \mu \mathrm{m}$, it does not have a large effect on the stimulus transfer, and its effect on the sealing resistance is even smaller. However, when the thickness of the sealing gap is doubled from $d_{g}=10 \mathrm{~nm}$ to $d_{g}=20 \mathrm{~nm}$, both the stimulus transfer and the sealing resistance are reduced by a factor of two. The same effect appears when the sealing gap is halved from $d_{g}=10 \mathrm{~nm}$ to $d_{g}=5$ $\mathrm{nm}$ : both the stimulus transfer and the seal- ing resistance are doubled, regardless of the size of the neuron.

\section{Finite Element Model versus Circuit Model}

As stated at the start, finite element modeling is proposed in this article as an alternative for lumped circuit modeling. In order to make a comparison between these two modeling methods, the parametrical geometry of the neuron-electrode interface is also used for computation of the components of a lumped circuit. Just as in the finite element model, only resistive membrane properties are modeled. Furthermore, since current stimulation is used, the electrode impedance is left out of the model.

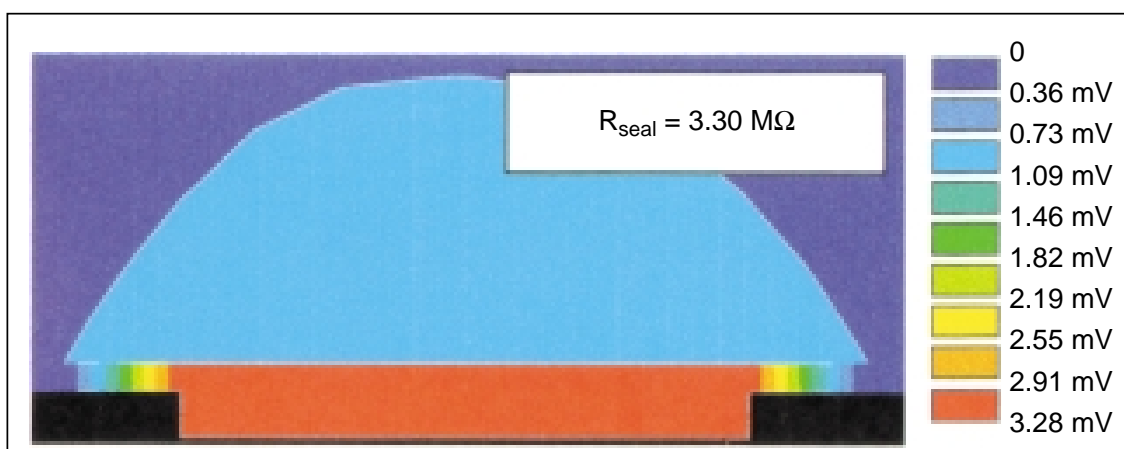

(a)

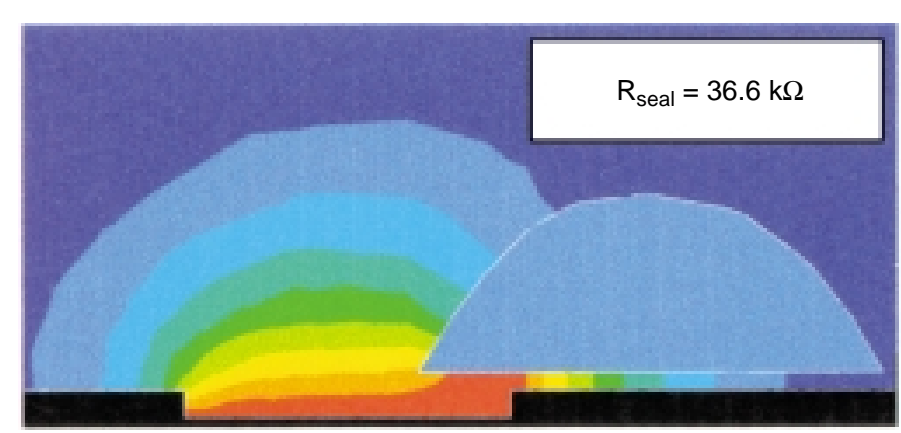

0

$4.06 \mu \mathrm{V}$ $8.13 \mu \mathrm{V}$ $12.2 \mu \mathrm{V}$ $16.3 \mu \mathrm{V}$ $20.3 \mu \mathrm{V}$ $24.4 \mu \mathrm{V}$ $28.5 \mu \mathrm{V}$ $32.5 \mu \mathrm{V}$ $36.6 \mu \mathrm{V}$

(b)

5. Computed potentials due to a $1 \mathrm{nA}$ stimulus current through the microelectrode in a cross section of the model. The equipotential lines are marked by the boundaries between two colored regions.

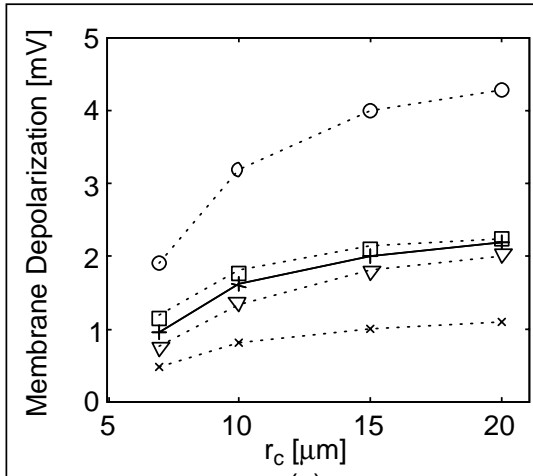

(a)

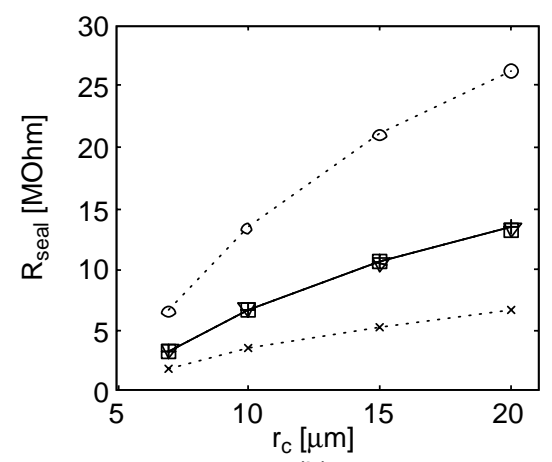

(b)

6. Effect of variation of several model parameters on upper membrane depolarization and on sealing resistance. The results from simulations with the initial parameter values $\left(h_{c}=5 \mu \mathrm{m}, d_{g}=10 \mathrm{~nm}\right)$ are plotted with a solid line. The dashed lines represent results from simulations in which one of the parameters is varied. (a) Effect of variation of the height of the cell $\left(\nabla h_{c}=1 \mu \mathrm{m}, \square h_{c}=9 \mu \mathrm{m}\right)$ and the thickness of the sealing gap $\left(0 d_{g}=5 \mathrm{~nm}, \times d_{g}=20 \mathrm{~nm}\right)$ on the upper membrane depolarization. (b) Effect of variation of the height of the cell $\left(\nabla h_{c}=1 \mu \mathrm{m}, \square h_{c}=9 \mu \mathrm{m}\right)$ and the thickness of the sealing gap $\left(0 d_{g}=5 \mathrm{~nm}, \times d_{g}=20 \mathrm{~nm}\right)$ on the sealing resistance. 
The resulting circuit model consists of three resistive components: the sealing resistance and the upper and lower membrane resistances (Fig. 7).

The depolarization of the upper membrane due to a stimulus current through the electrode can be written as:

When the neuron is centered on the electrode $\left(x_{c}=0\right)$, the geometry parameters can be used for computing the circuit components:

$$
\begin{gathered}
R_{\text {seal }}=\frac{1}{2 \pi \cdot d_{g} \cdot \sigma_{\text {medium }}} \ln \left(\frac{r_{c}}{r_{e}}\right) \\
R_{\text {low }}=\frac{1}{\pi \cdot \sigma_{\text {mem }} \cdot r_{l}^{2}}
\end{gathered}
$$

$$
V_{\text {up }}=R_{\text {seal }} \frac{R_{\text {up }}}{R_{\text {seal }}+R_{\text {low }}+R_{\text {up }}} \cdot I_{\text {stim }}
$$

$$
R_{\text {up }}=\frac{1}{\pi \cdot \sigma_{\text {mem }} \cdot f\left(h_{c}\right) \cdot r_{c}^{2}}
$$

with the radius of the lower membrane $r_{l}=r_{e}$ and $f\left(h_{c}\right)=1.43$, a correction factor for the area of the upper membrane, due to the height of the neuron.

An interesting test case for the comparison between finite element modeling and lumped circuit modeling is variation of the radius of the electrode. The electrode radius is a design parameter of the MEA and can be adjusted for optimization of the neuron-electrode contact. A decrease of the radius of the electrode $r_{e}$ will have two contradictory effects on the stimulus transfer: On one hand, the length of the current path through the sealing gap is increased, thereby increasing the sealing resistance and the stimulus transfer. On the other hand, the area of the lower mem-

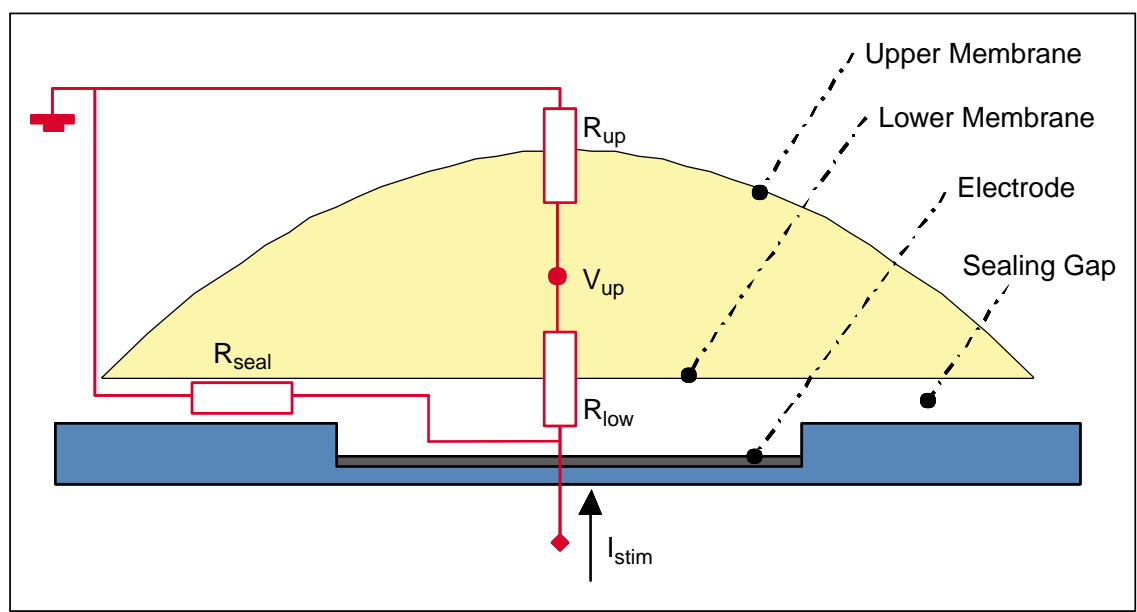

7. Circuit model of the neuron-electrode interface, used for comparison with the finite element model. The electrode impedance is left out of the model and only resistive membrane properties are modeled. All component values are computed from the geometry parameters (Fig. 3).

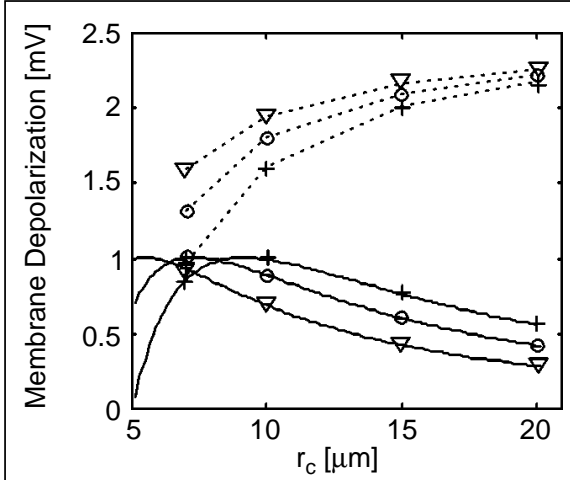

(a)

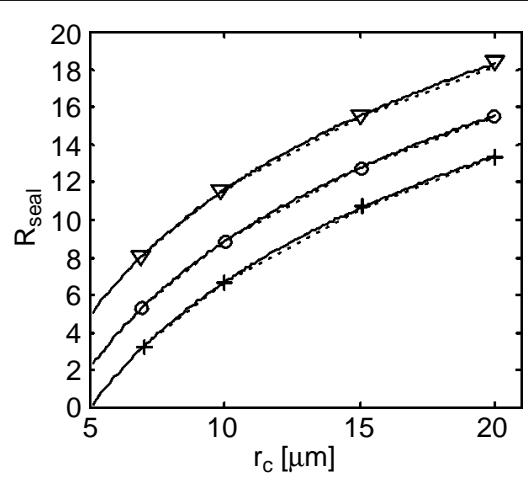

(b)
8. Comparison between simulation results from circuit model (solid lines) and finite element model (dashed lines). (a) Upper membrane depolarization, plotted versus the radius of the neuron while the radius of the electrode is varied $\left(+r_{e}=5 \mu \mathrm{m}, o\right.$ $r_{e}=4 \mu \mathrm{m}, \nabla r_{e}=3 \mu \mathrm{m}$ ) (b) Sealing resistance, plotted versus the radius of the neuron while the radius of the electrode is varied $\left(+r_{e}=5 \mu \mathrm{m}, 0 r_{e}=4 \mu \mathrm{m}, \nabla r_{e}=3 \mu \mathrm{m}\right)$. brane (covering the electrode surface) is decreased, thereby decreasing the current through the cell and, hence, the stimulus transfer. Depending on which of these two effects is the strongest, the stimulus transfer may increase or decrease with decreasing electrode radius. Also, an optimal electrode radius may exist with respect to the stimulus transfer. Obviously, the net effect of a decreasing electrode radius on the stimulus transfer must be obtained by proper modeling of the neuron-electrode interface, which makes this an ideal test case for a comparison between the two modeling methods.

In Fig. 8, the stimulus transfer and sealing resistance, as computed with both the finite element model and the circuit model, are plotted versus the radius of the neuron. In these simulations, the electrode radius is decreased in three steps from $r_{e}=5 \mu \mathrm{m}$ to $r_{e}=3 \mu \mathrm{m}$. For the stimulus transfer, there appears to be a striking difference between the two models [(Fig. 8(a)]. The results of the circuit model suggest that an optimal stimulus transfer exists for a certain radius of the neuron, and that this optimum depends on the size of the electrode. The results from the finite element model, however, do not show this optimum. Instead, the finite element model predicts a higher stimulus transfer.

The results in Fig. 8(b) show that this disagreement cannot be explained by differences in the computed sealing resistance. Instead, it arises from the membrane over the sealing gap, which is not represented in the circuit model. Due to the potential drop that exists over this sealing membrane, a radially decreasing membrane current arises from the sealing gap into the intracellular space (Fig. 9). This current is negligible compared to the leakage current in the sealing gap and will not affect the computation of the sealing resistance. However, it is not negligible compared to the current through the part of the lower membrane that covers the electrode. Hence, this membrane current contributes significantly to the stimulus transfer.

\section{Nonparametrical Geometry}

The finite element method permits modeling of almost any geometry and is not restricted to parametrical geometries, as defined above. Therefore, more realistic shapes of neurons can be modeled easily, which is very advantageous when it comes to experimental characterization of the electrical properties of the neuron-electrode interface. As an illustration, 
the two examples of neuron-electrode interfaces from the beginning of this article [Figs. 1(b), Fig. 1(d)] are used for reconstruction of the interface geometry. The circumferences of neuronal soma and electrode are marked on a digitized microphotograph. From these contours, the geometries are reconstructed [Fig. 10(a)]. The assumption of a parabolic height profile is applied again, since the above presented simulations revealed that the influence of height was not very large. The maximum height was taken as a quarter of the maximal diameter of the neuron.

Based on the reconstructed geometry, mesh generation is performed in ANSYS. Again, a stimulus current is applied through the electrode, and the membrane potentials are computed. In Fig. 10(b), the membrane potential distribution is de- picted on the model that is superposed on an image of the electrode. A view, from below, in Fig. 10(c), reveals that most of the membrane polarization is localized at the site of electrode and in the sealing gap. The computed potentials can be related directly to experimentally derived potentials of the electrode and the intracellular space, which permit identification of the electrical properties of the neuron-electrode interface.

\section{Conclusion}

The finite element model offers several advantages above lumped circuit modeling. It permits linking of the electrical properties of the neuron-electrode interface to a variety of more or less complex geometries, including cases of both complete and defective sealing. All

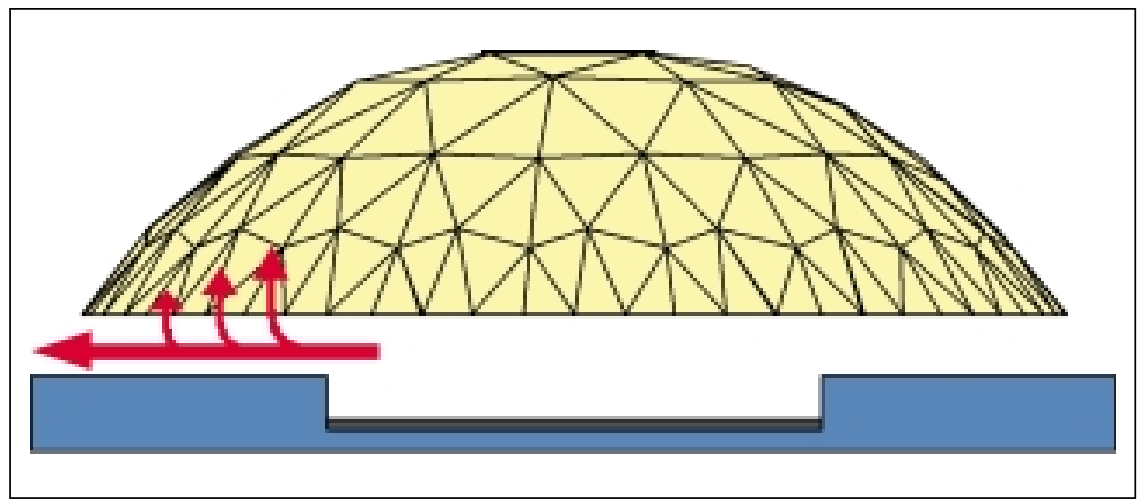

9. The difference between the circuit model and the finite element model is caused by the current that flows from the sealing gap to the intracellular space, due to a radially decreasing potential drop over the sealing part of the neuronal membrane. In the lumped circuits this current is not modeled. The finite element model accounts inherently for this current.

currents and potentials in the neuron-electrode interface are computed inherently by generating a numerical solution of the finite element problem, which obviates the need for simplifying the electrical representation of the geometry. Furthermore, this model can be easily extended with the implementation of active membrane properties and axons, which allow simulation of extracellular stimulation as well as recording.

The applications of this modeling method are numerous. For example, using geometrical data it can be used for electrical characterization of the sealing gap and the effects of several adhesion promoters on sealing resistance. It also permits modeling of the influence of local (active) membrane properties, such as ion-specific channel densities, on extracellular recording and stimulation. Finally, it would greatly facilitate the optimization of the MEA substrate and electrode geometries for improved neuron-electrode contact.

\section{Acknowledgment}

This work was supported by the BIOMED II EC project, shared cost contract no. BMH4-2723.

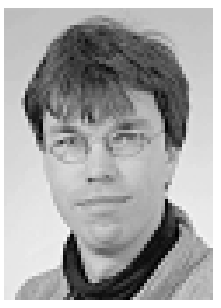

Jan Reinoud Buitenweg received his M.Sc. in electrical engineering in 1998 at the University of Twente. He is currently working there as a Ph.D. student in the Signals and Systems group of the Faculty of
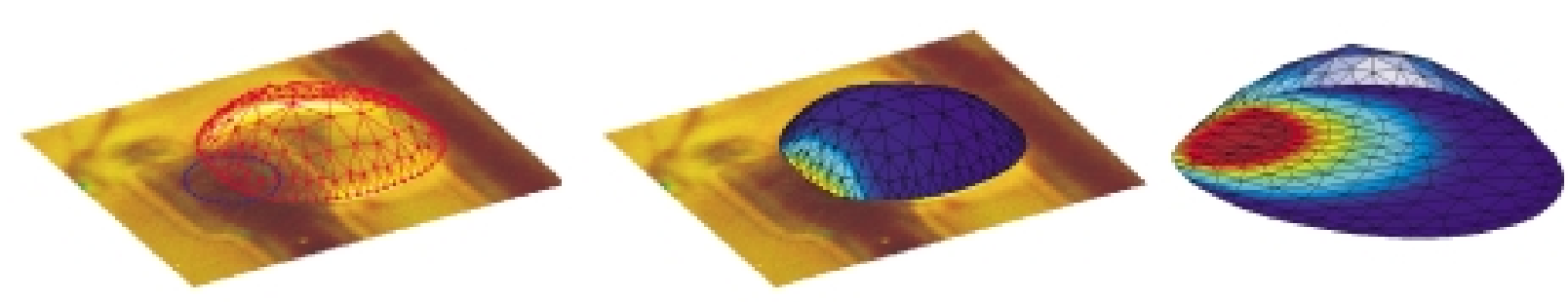

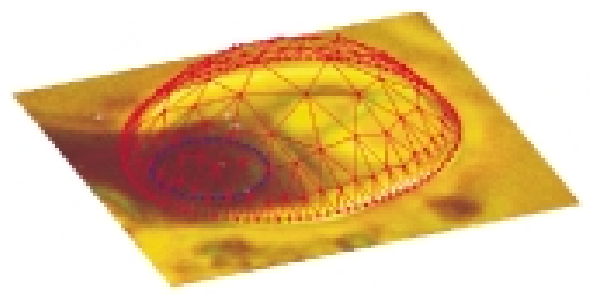

(a)

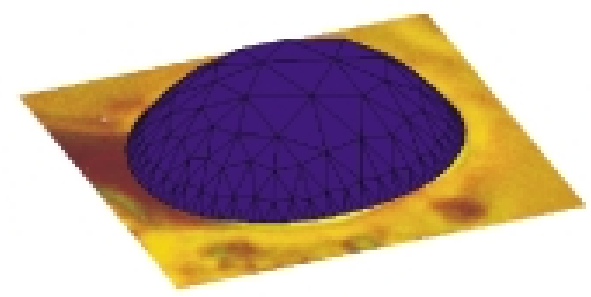

(b)

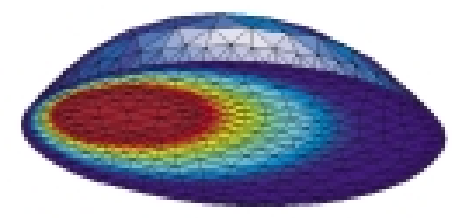

10. Modeling of neuron-electrode interfaces using geometry reconstruction from microphotographs. The pseudocolors mark the equipotential lines over the membrane (not to scale). 
Electrical Engineering. His research interests include the characterization and measurement of the electrical contact between cultured neurons and embedded microelectrodes.

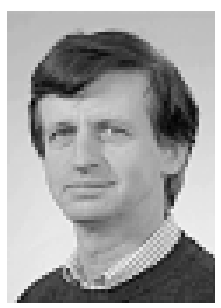

Wim Rutten was trained as an experimental physicist (Ph.D. degree in 1979) at Leiden University. Thereafter he studied the auditory system at the ENT Department of Leiden University Hospital. Since 1985 he has been with the BMTI/Faculty of Electrical Engineering of the University of Twente, as assistant and associate professor of Biomedical Control Systems. His present research interests are neurotechnology (neuro-electronic interfaces,cultured probes), signal processing, and bioelectricity.

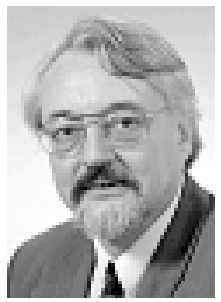

Enrico Marani was trained as a neuroanatomist $(\mathrm{PhD}$ degree 1982) at Leiden University. Thereafter he became head of the Neuroregulation Group at the Department of
Physiology, Leiden University. Since 1997 he has been a part-time professor in neurophysiology at the BMTI/Faculty of Electrical Engineering of the University of Twente. His present research interests are neuroregulation and neurotechnology, especially in the field of nerve regeneration.

Address for Correspondence: J.R. Buitenweg, Signals and Systems Group, Faculty of Electrical Engineering, University of Twente, P.O. Box 217, 7500 AE Enschede, The Netherlands. Tel. +31 53 489 2759. Fax. +31 53489 2287. E-mail: J.R.Buitenweg@el.utwente.nl

\section{References}

1. Rutten WLC, Smit JPA, Frieswijk TA, Bielen JA, Brouwer ALH, et al.: Neuro-electronic interfacing with multielectrode arrays: Selectivity and efficiency of motor-fiber stimulation, toward a cultured probe. IEEE Eng Med Biol Mag 18(3): 47-55, 1999.

2. Jimbo Y, Robinson HPC, Kawana A: Strengthening of synchrinized activity by tetanic stimulation in cortical cultures: Application of planar electrode arrays. IEEE Trans Biomed Eng 45(11): 1297-1304, 1998.

3. van Pelt J, Wolters P, van Veen D, Bomer J, Rutten WLC, et al.: Long-term multielectrode registration of neuronal firing activity from rat ce- rebral cortex tissue in vitro. In: Proc Int Conf IEEE Eng Med \& Biol Soc (CDROM), 1996.

4. Maher MP, Pine J, Wright J, and Tai YC: The neurochip: A new multielectrode device for stimulating and recording from cultured neurons. J Neurosc Meth 87: 45-56, 1999

5. Gross GW: Simultaneous single unit recording in vitro with a photoetched laser deinsulated gold multimicroelectrode surface. IEEE Trans Biomed Eng BME-26(5): 273-279, 1979.

6. Regehr WG, Pine J, Cohan CS, Mischke MD, and Tank DW: Sealing cultured invertebrate neurons to embedded dish electrodes facilitates long-term electrical stimulation and recording. J Neurosci Meth 30: 91-106, 1989.

7. Bove M, Grattarola M, Martinoia S, and Verreschi G: Interfacing cultured neurons to planar substrate microelectrodes: Characterisation of the neuron-to-microelectrode junction Bioel Chem and Bioen 38: 255-265, 1995.

8. Biological Handbooks of the Federation of the American Societies for Experimental Biology: Biological Databooks I and II, 1974.

9. Biological Handbooks of the Federation of the American Societies for Experimental Biology: Growth, 1962.

10. Ziehen TH: Bardenleben's Handbuch des Anatomie IV 1-2, Das Nervensystem, Jena, Gustav Fischer Verlag, 1903.

11. Gingell D: Cell contact with solid surfaces. In: Glaser R and Gingell D (Eds.): Biophysics of the Cell Surface. New York: Springer-Verlag, pp. 263-286, 1990. 